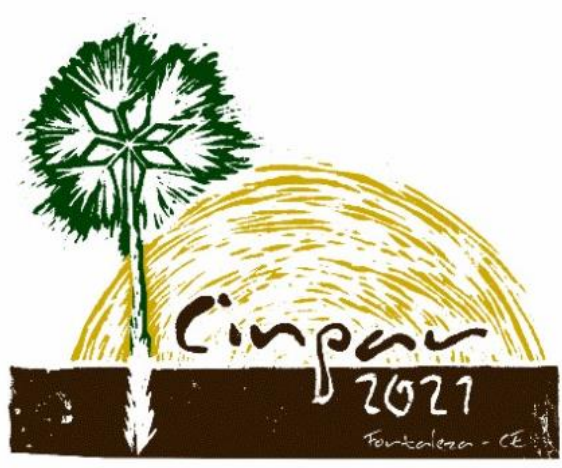

XVII Congresso Internacional sobre Patologia e

Reabilitação das Construções

XVII Congreso Internacional sobre Patología y Rehabilitación de las Construcciones

XVII International Conference on Pathology and Constructions Rehabilitation

FORTALEZA (Brasil), 3 a 5 de junho de 2021

https://doi.org/10.4322/CINPAR.2021.077

\title{
Análise da relevância das características dos revestimentos cerâmicos de fachada na sua degradação
}

\section{Analysis of the relevance of characteristics in the façade degradation process}

\author{
Alan Ribeiro SANTOS ${ }^{1}$, Jéssica Siqueira de SOUZA ${ }^{2}$, Ana SILVA ${ }^{3}$, Suzanna Ferreira PONTES ${ }^{4}$
}

\author{
${ }^{1}$ Universidade de Brasília, Brasília, Brasil, alanrsmbs@gmail.com \\ 2 Universidade de Brasília, Brasília, Brasil, jss.siqueira@gmail.com \\ 3 Universidade de Lisboa, Lisboa, Portugal, anasilva931@msn.com \\ ${ }^{4}$ Universidade de Brasília, Brasília, Brasil, suzanna.pontes14@hotmail.com
}

\begin{abstract}
Resumo: O desempenho de um sistema de revestimento cerâmico de fachadas, que é dependente de uma série de fatores distintos, é de fundamental importância para a proteção adequada de edifícios. Os diversos agentes envolvidos no processo de degradação podem ser de diferentes naturezas. A durabilidade pode ser afetada em níveis variados e com características únicas capazes de gerar danos de maneira heterogênea e consequentemente influenciando de forma particular a vida útil do sistema. Esta pesquisa busca identificar a relevância de variáveis analisadas no processo de degradação de sistemas de revestimento cerâmico em fachadas. Métodos estatísticos foram utilizados para a definição do grau de importância das variáveis estudadas no processo de degradação das fachadas revestidas por cerâmicas de edifícios na cidade de Brasília. O nível da degradação foi mensurado a partir do indicador de degradação denominado Fator Geral de Degradação. A análise estatística apontou forte correlação entre a idade do edifício, dimensão e cor predominante das pastilhas e o indicador de degradação. Os resultados obtidos são de grande valor para a construção de modelos eficientes de previsão de vida útil de sistemas de revestimento cerâmico de fachadas, além de poderem ser utilizados para a orientação de intervenções, investimentos em fases de projeto e execução, entre outros.
\end{abstract}

Palavras-chave: Durabilidade; Degradação; Fachada; Revestimento cerâmico.

Abstract: The performance of a ceramic cladding system in façades, which is dependent on several different factors, is crucial for the proper protection of buildings. The different agents involved in the degradation process can have different natures. The durability of ceramic claddings can be affected at varying levels and with unique characteristics capable of causing damage in a heterogeneous manner and, consequently, influencing in a particular way the system's service life. This study intends to identify the relevance of the variables analyzed in the degradation process of ceramic cladding systems on facades. Statistical methods were used to define the degree of importance of the variables studied in the process of degradation of façades covered by ceramic claddings in the city of Brasília. The level of degradation was measured using the degradation index called General Degradation Factor. The statistical analysis showed a strong correlation between the age of the building, the size and color of the tiles and the degradation index. The results obtained are extremely relevant for the construction of efficient models for predicting the service life of ceramic cladding systems in facades, in addition to being able to be used to guide interventions, investments in design and execution phases, among others.

Keywords: Durability; Degradation; Facade; Ceramic Cladding. 


\section{Introdução}

Nas últimas décadas, sobretudo devido a fatores econômicos e ambientais, a demanda por um conhecimento pormenorizado do processo de degradação dos edifícios e suas partes cresceu significativamente. A mesma necessidade tem se estendido para o sistema utilizado no revestimento das fachadas, pois esse exerce um papel importante na proteção e desempenho das edificações. Os sistemas de revestimento cerâmico de fachadas apresentam diversas vantagens, desde aspectos estéticos a facilidade de limpeza. Entretanto, é natural que com o decorrer do tempo haja o surgimento de anomalias oriundas de diversas causas.

Características variadas em um sistema de revestimento cerâmico interferem em seu processo de degradação. Shohet e Laufer (1996) apontam, por exemplo, a elevada sensibilidade do sistema à qualidade dos materiais, da aplicação, da mão-de-obra, do projeto e da regularidade do substrato. Ao passo que Lacasse (2003) destaca o efeito dos agentes climáticos para deterioração do sistema e Souza et al. (2018a) salienta a combinação de vários fatores para o surgimento de anomalias.

Bauer et al. (2014) apontam, em ordem de maior ocorrência, descolamento cerâmico, fissuração, falha nas juntas (falha no rejunte) e eflorescência como algumas das manifestações patológicas mais comuns em revestimentos cerâmicos de fachadas (Figura 1). Essas anomalias evoluem de diferentes maneiras de acordo com as características de implementação e condição do revestimento, dependentes da orientação da fachada, da idade, do elemento construtivo, da dimensão da cerâmica, entre outros (Souza et al., 2018b).

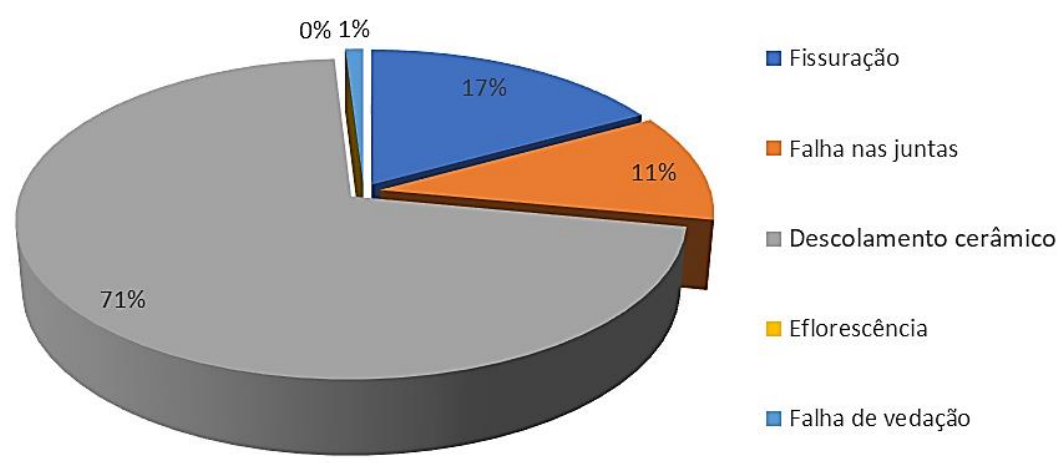

Figura 1- Anomalias comuns em sistemas de revestimento cerâmico (adaptada de Bauer et al., 2014)

Tendo em vista a grande variabilidade de condições de materiais, projeto e exposição ambiental de um sistema de revestimento cerâmico de fachada, o presente trabalho busca identificar as principais características relevantes para o processo de degradação. Além de entender como a combinação dessas características podem propiciar o surgimento de anomalias ao longo do tempo, a fim de possibilitar a orientação de projetos. Para tal, dados de degradação de fachadas de 46 edifícios localizados na região Centro-oeste do Brasil foram analisados. O nível de degradação foi definido a partir do indicador de degradação denominado Fator Geral de Degradação (FGD), sendo esse, em seguida, avaliado quanto a sua correlação com as características do sistema de revestimento cerâmico. Ademais, analisou-se o surgimento das anomalias mais comumente associadas ao sistema em relação à combinação da orientação da fachada e as características de materiais.

\section{Metodologia}

A degradação dos edifícios analisados foi mensurada a partir do Método de Mensuração de Degradação (MMD). Esta metodologia foi desenvolvida por pesquisadores da Universidade de Brasília (Bauer et al., 2020) e expressa quantitativamente a degradação de fachadas de edifícios por meio de inspeções visuais das fachadas e mapeamento dos danos. Dentre os indicadores de degradação apresentados pelo MMD, o FGD foi o indicador adotado para a análise das variáveis no processo de degradação. Esse indicador pode ser obtido pela equação 1 . 


$$
F G D=\frac{\sum\left(A_{d(n)} \cdot k_{n} \cdot k_{c(n)}\right)}{A \cdot \sum k_{\text {máx }}}
$$

Em que $F G D$ é o Fator Geral de Danos, $A_{d(n)}$ é a área de fachada afetada por $\mathrm{n}$ tipos de anomalias, $k_{n}$ é o nível de condição das anomalias (de 1 a 4), $k_{c(n)}$ é a importância relativa das anomalias, $k_{\text {máx é a constante }}$ equivalente à pior condição combinada de todas as anomalias $\left(k_{\text {máx }}=14\right), A$ é a área total da fachada e $n$ é a referência do tipo de anomalia que varia de 1 a $4(n=1$ : descolamento cerâmico, $n=2$ : fissuração, $n=3$ : falha nas juntas e $n=4$ : eflorescência).

Além da mensuração da degradação consolidada por meio do FGD, foram coletadas para cada fachada características de projeto, dos materiais e das condições de exposição, sendo esses os atributos analisados. As características consideradas refletem a idade, o elemento construtivo, a claridade (cor) da pastilha, a dimensão da pastilha, a existência de juntas horizontais e a orientação da fachada. Baseado nos resultados encontrados por Souza (2019), os critérios para classificação e a quantificação destas variáveis estão apresentados no Quadro 1. Ressalta-se que esta quantificação foi apoiada nos valores sugeridos pela ISO 15686-7 (2012). O processo de quantificação diferencia-se do feito em Souza (2019) haja vista a correlação das características estudadas com o FGD ao invés da vida útil estimada da amostra. Tendo-se ainda que as variáveis correspondentes a claridade da cerâmica clara e presença de juntas recebem valores diferentes dos resultados diretos obtidos devido ao entendimento que essas contribuem negativamente para a degradação da amostra, implicando consequentemente em um menor indicador de degradação.

Quadro 1- Critérios para classificação e quantificação das variáveis

\begin{tabular}{|c|c|c|}
\hline Variáveis & Classificação e critérios & Valor \\
\hline Idade & Conforme a idade & - \\
\hline Elemento & $\begin{array}{l}\text { - Fachada } \\
\text { - Empena } \\
\text { - Caixa de escada }\end{array}$ & $\begin{array}{l}1,00 \\
0,94 \\
1,00\end{array}$ \\
\hline Claridade & $\begin{array}{l}\text { - Clara: absortância predominante menor que } 30 \% \\
\text { - Não clara: absortância predominante maior que } 30 \%\end{array}$ & $\begin{array}{l}0,90 \\
0,97\end{array}$ \\
\hline Dimensão & $\begin{array}{l}\text { - Pequena: cerâmica com área menor que } 4 \mathrm{~cm}^{2} \\
\text { - Grande: cerâmica com área maior que } 4 \mathrm{~cm}^{2}\end{array}$ & $\begin{array}{l}0,97 \\
1,04\end{array}$ \\
\hline Juntas & $\begin{array}{l}\text { - Presença: Se houver existência de juntas horizontais } \\
\text { - Ausência: Se não houver existência de juntas horizontais }\end{array}$ & $\begin{array}{l}0,90 \\
1,00\end{array}$ \\
\hline Orientação & $\begin{array}{l}\text { - Norte: Azimute entre } 315^{\circ} \text { e } 44^{\circ} \\
\text { - Leste: Azimute entre } 45^{\circ} \text { e } 134^{\circ} \\
\text { - Sul: Azimute entre } 135^{\circ} \text { e } 224^{\circ} \\
\text { - Oeste: Azimute entre } 225^{\circ} \text { e } 314^{\circ}\end{array}$ & $\begin{array}{l}1,16 \\
0,85 \\
1,12 \\
0,88\end{array}$ \\
\hline
\end{tabular}

Para a determinação das características mais correlacionadas com o $F G D$ das fachadas, foi feita uma análise de correlação bivariada com teste de significância bilateral dada a natureza da hipótese testada, com auxílio do software IBM SPSS. Além disso, os coeficientes de correlação de Spearman e Kendall (tau-b de Kendall) foram utilizados. Essas correlações são não paramétricas haja vista a não normalidade de distribuição dos dados analisados conforme apontado pelos testes de normalidade de Kolmogorov-Smirnov e Shapiro-Wilk realizados também com auxílio do SPSS.

Por fim, o surgimento das anomalias mais comuns ao longo do tempo, de acordo com a orientação das fachadas e características dos materiais das fachadas, foi analisado. Para tal, a porcentagem de área degradada da fachada foi estudada para cada tipo de anomalia (descolamento cerâmico, fissuração, falha nas juntas e eflorescência). Após a consolidação das análises isoladas, as porcentagens de áreas degradadas 
para algumas anomalias principais, a orientação da fachada e a característica estudada foram verificadas ao longo do tempo permitindo maior compreensão da evolução das anomalias.

\section{Análise de resultados}

\subsection{Teste de correlação}

As correlações não paramétricas por meio dos coeficientes de correlação de Spearman e Kendall (tau-b de Kendall) foram observadas. Em ambas as análises todas as variáveis testadas (idade, cor da pastilha, dimensão da pastilha, elemento construtivo, orientação e existência de juntas) apresentaram, para o teste de significância bilateral, correlação significativa no nível 0,01 com o Fator Geral de Degradação (FGD). Os valores dos coeficientes de correlação obtidos em relação ao $F G D$ para os dados analisados estão apresentados no Quadro 2. A representação gráfica dos valores absolutos encontrados dos coeficientes para as diferentes variáveis analisadas estão ilustradas na Figura 2.

Quadro 2 - Coeficientes de correlação

\begin{tabular}{|c|c|c|c|c|c|c|c|}
\hline \multicolumn{2}{|c|}{$\begin{array}{c}\text { Correlações não } \\
\text { paramétricas } \\
\text { (em relação ao } F G D \text { ) }\end{array}$} & Idade & Claridade & Dimensão & Elemento & Orientação & Juntas \\
\hline \multirow{2}{*}{ Kendall } & $\tau_{B}$ & 0,488 & $-0,395$ & $-0,260$ & 0,212 & 0,199 & 0,190 \\
\cline { 2 - 9 } & Valor-p & $9,6 \mathrm{E}-39$ & $3,8 \mathrm{E}-19$ & $3,8 \mathrm{E}-09$ & $1,5 \mathrm{E}-06$ & $7,8 \mathrm{E}-07$ & $1,7 \mathrm{E}-05$ \\
\hline \multirow{2}{*}{ Spearman } & $\rho$ & 0,673 & $-0,482$ & $-0,318$ & 0,260 & 0,268 & 0,232 \\
\cline { 2 - 8 } & Valor-p & $6,6 \mathrm{E}-47$ & $1,7 \mathrm{E}-21$ & $1,6 \mathrm{E}-09$ & $1,0 \mathrm{E}-06$ & $4,5 \mathrm{E}-07$ & $1,4 \mathrm{E}-05$ \\
\hline
\end{tabular}

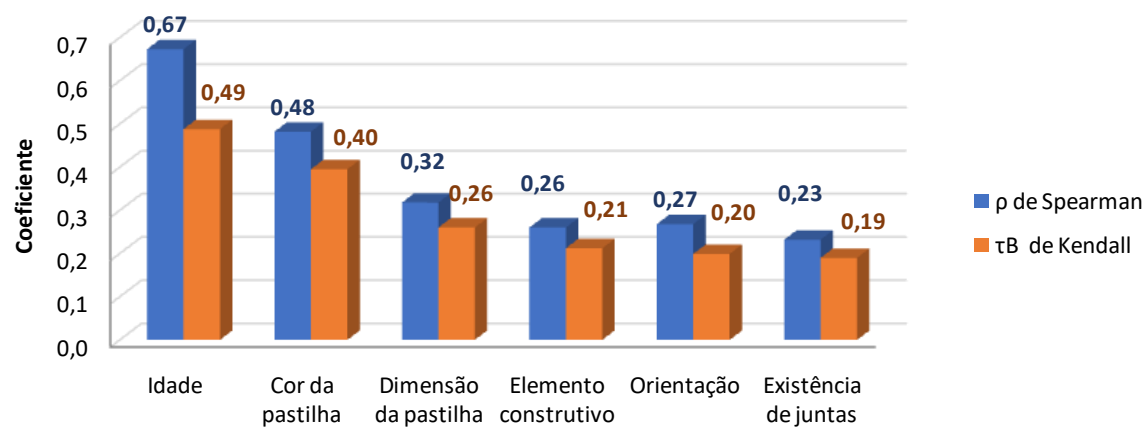

Figura 2 - Valores absolutos dos coeficientes de correlação

Os resultados demonstraram que a variável idade é a mais fortemente correlacionada com o FGD, conforme amplamente apontado pela literatura (Souza, 2016) e intuitivamente inferido. Seguida pela variável claridade da pastilha, com valores negativos dos coeficientes de correlação, provavelmente devido ao fato de cerâmicas de cores claras estarem presentes em fachadas mais antigas, naturalmente associadas a maiores degradações. De acordo com Uchôa (2015) e Moscoso (2013), para as mesmas condições de insolação, cerâmicas de cores mais escuras absorvem mais radiação solar, implicando no alcance de maiores temperaturas e consequentemente maiores tensões e deformações comparadas às cerâmicas claras. Daniotti e Re Cecconi (2010), por sua vez, apontam as grandes variações associadas às diferentes cores de um material em seu processo de degradação.

Após a variável cor da pastilha, segue como mais fortemente correlacionada com o FGD a variável dimensão da pastilha, sendo os valores negativos dos coeficientes de correlação explicados por motivo da inexistência de cerâmicas grandes nas fachadas mais antigas. Segundo Uchôa (2015), placas cerâmicas de menor dimensão estão associadas a maior estabilidade do sistema de revestimento haja vista a maior quantidade de juntas existentes que permitem uma dissipação mais eficiente das tensões internas. Concomitantemente tem-se também que materiais de menor dimensão estão sujeitos a menores 
dilatações térmicas. Assim sendo, há menores tensões associadas à deformação, vide a proporcionalidade direta existente entre a dilatação do material e sua dimensão (James et al., 2001).

Em relação à correlação da orientação e do elemento construtivo, a depender do coeficiente de correlação observado, esses apresentaram ordens distintas de força de relação com o FGD. Segundo Souza (2019), os processos de degradação para os distintos elementos construtivos ocorrem em ritmos de aceleração diferentes. Tratando-se da orientação, as orientações com maior suscetiblidade à degradação, ordenadamente, são Norte, Sul, Oeste e Leste (Francinete et al., 2015; Souza, 2016). Esses resultados apontam a fachada Norte como a mais suscetível à degradação, fato explicado tendo em vista a alta incidência de chuva e radiação solar sobre a mesma. Seguida pela fachada Sul, resultado que pode ser justificado pelo fato de fachadas orientadas para ao Sul no hemisfério sul, mesmo exposta a menores cargas térmicas durante $o$ ano, receberem as maiores taxas de radiação solar durante o Verão, época mais quente do ano (Corbella e Yannas, 2009; Braga e Amorim, 2004).

Em último lugar, em se tratando de força de correlação com o $F G D$, está a variável existência de juntas. Ribeiro e Barros (2010) e Souza et al. (2018a) destacam a importância da presença de juntas para minimização da propagação de esforços frente as deformações decorrentes da ação dos agentes climáticos. Essas são fundamentais para se evitar manifestações patológicas no sistema de revestimento cerâmico e, por conseguinte, aumentar sua vida útil. É importante ressaltar que somente as fachadas mais novas apresentavam juntas, levando a dificuldades na análise e determinação de valores inconformes.

Diante do exposto, nota-se que após a variável idade, as características dos materiais (cor e dimensão da pastilha) são as que apresentaram maior correlação com a degradação do sistema de revestimento cerâmico. Embora tenha que se ter cautela frente à grande complexidade e interseccionalidade das variáveis testadas, tem-se que os resultados apontam para grande sensibilidade do sistema à inadequação dos materiais. Esses resultados convergem com as afirmações de Shohet e Laufer (1996) e Bordalo et al. (2010), que realçam a importância da etapa de projeto para o desempenho adequado do sistema de revestimento cerâmico de fachadas.

\subsection{Relação das anomalias com as características}

Para a análise do surgimento de anomalias ao longo do tempo relacionadas à orientação da fachada e às características dos materiais, foram utilizadas as fachadas com idades de no máximo 35 anos, pois a partir dessa idade ou próximo disso todas as amostras eram pequenas e de cor clara, fato que poderia prejudicar a análise já que essas, naturalmente, apresentariam linhas de tendência indicando maior degradação. As características de materiais analisadas foram claridade e dimensão da pastilha, sendo examinado o surgimento de algumas manifestações patológicas comuns e a orientação.

Uma vez que há uma elevada incidência e considerando a alta gravidade associada ao descolamento cerâmico, a análise em relação à claridade da cerâmica foi feita em função dessa anomalia, assim propiciando uma investigação mais robusta. A Figura 3 ilustra a relação da porcentagem de área afetada ao longo do tempo em função da claridade da pastilha para as orientações. 


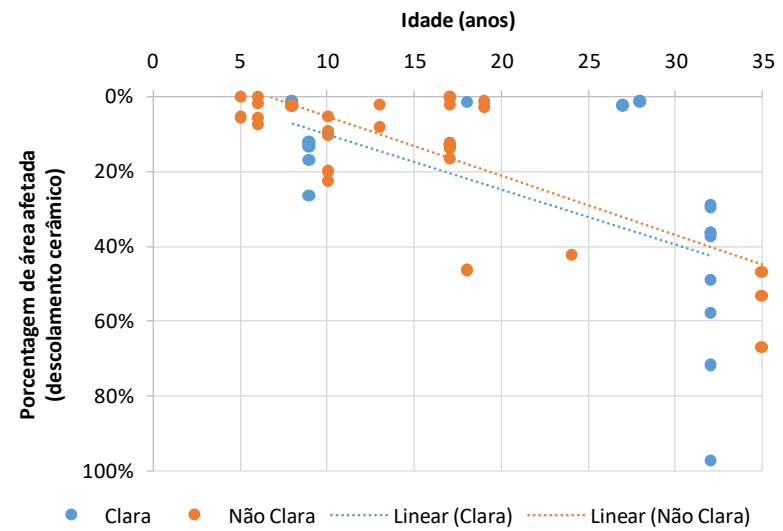

(a) Norte

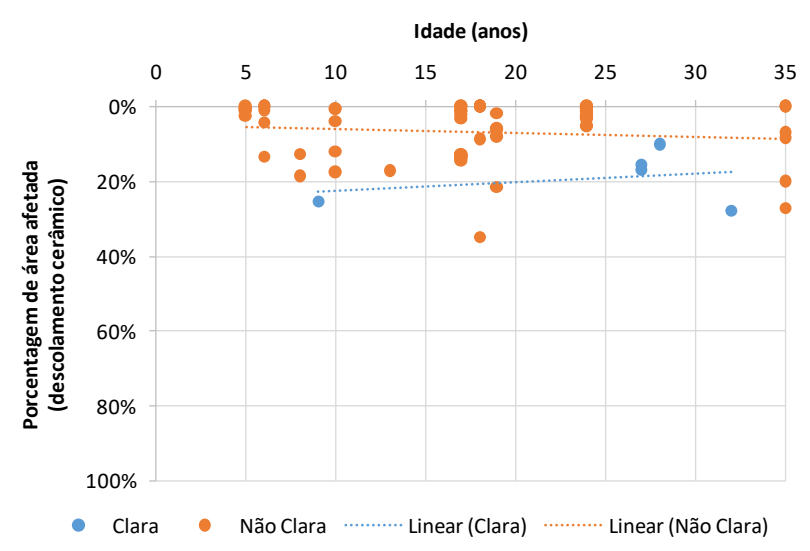

(c) Leste

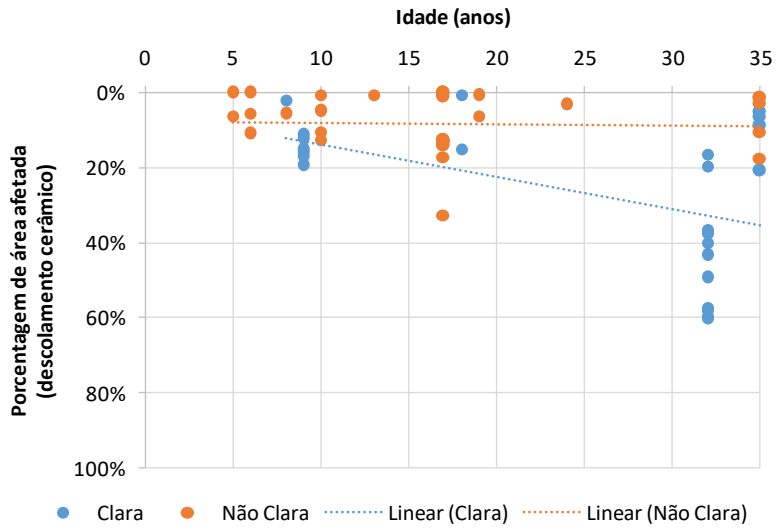

(b) Sul

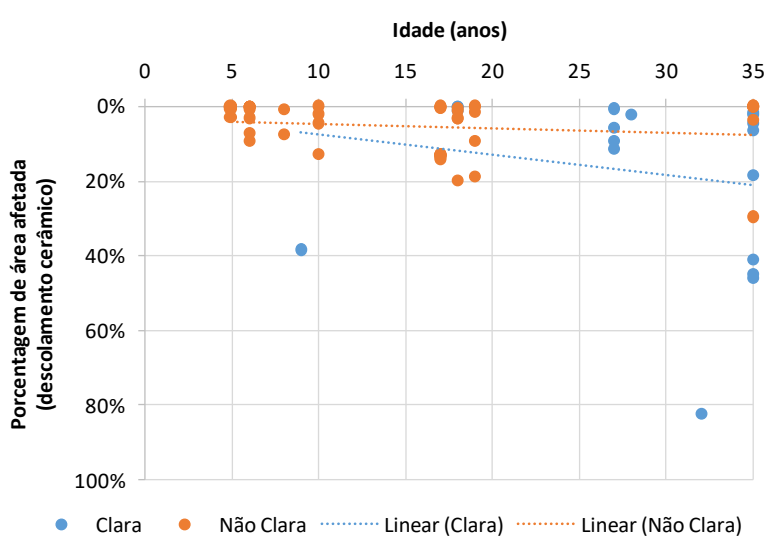

(d) Oeste

Figura 3 - Relação da porcentagem de área de descolamento cerâmico em função da claridade da pastilha para as orientações

Os resultados apontaram para as cerâmicas não claras com maiores porcentagens de área afetada por descolamento cerâmico nas fachadas Norte e Leste. É importante observar que, por mais que a análise da linha de tendência para as cerâmicas claras seja incerta devido à desproporcionalidade de dados em certas idades, os casos plotados de descolamento cerâmico em amostras não claras são mais volumosos e com valores maiores na maior parte do tempo. Para as fachadas orientadas ao Sul e a Oeste,as cerâmicas claras apresentaram maior porcentagem de área afetada por descolamento cerâmico.

As maiores porções de área afetada por descolamento cerâmico nas pastilhas não claras nas fachadas Norte e Leste podem ser explicadas pelo fato dessas orientações sofrerem maiores incidências de radiação solar ao longo do ano. Assim sendo, já que as cerâmicas não claras estão sujeitas a maiores tensões e deformações do que as cerâmicas claras para as mesmas condições de insolação (Uchôa, 2015; Moscoso, 2013), é esperado para as pastilhas não claras maior degradação.

Por sua vez, as maiores ocorrências de descolamento cerâmico em cerâmicas claras nas fachadas orientadas ao Sul e a Oeste podem ser justificadas pelo fato dessas orientações receberem menores cargas térmicas ao longo do ano. Desta forma, ao invés de se ter maiores degradações associadas a maiores taxas de absorção de radiação solar, o contrário acontece, onde as cerâmicas claras estarão submetidas a resfriamentos mais intensos, consequentemente retrações mais acentuadas, maiores tensões internas de compressão e maiores danos.

Em relação à dimensão da cerâmica, o surgimento de descolamento cerâmico e falha no rejunte foi verificado. A consideração do descolamento tem a mesma motivação da análise da claridade da pastilha. A investigação de falha nas juntas, por sua vez, é motivada pelo fato de cerâmicas com menores dimensões possuírem um maior número de juntas permitindo uma dissipação mais eficiente das tensões internas. A Figura 4 ilustra a relação da porcentagem de área afetada ao longo do tempo em função da dimensão das cerâmicas para as orientações. 


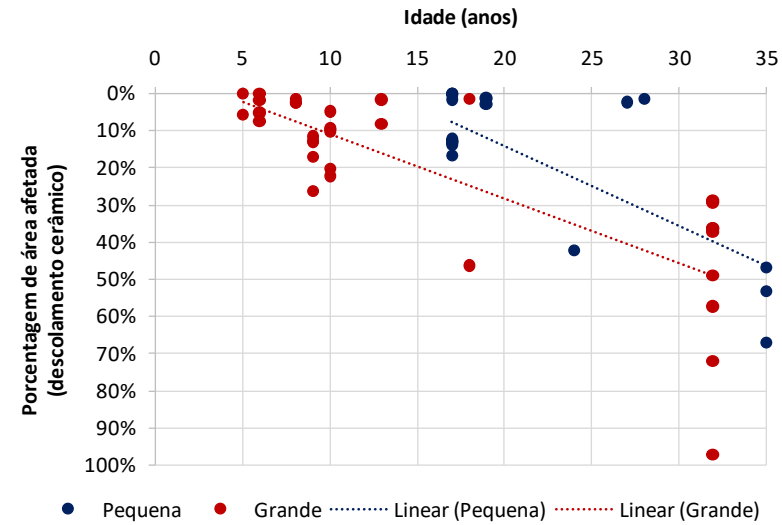

(a) Norte

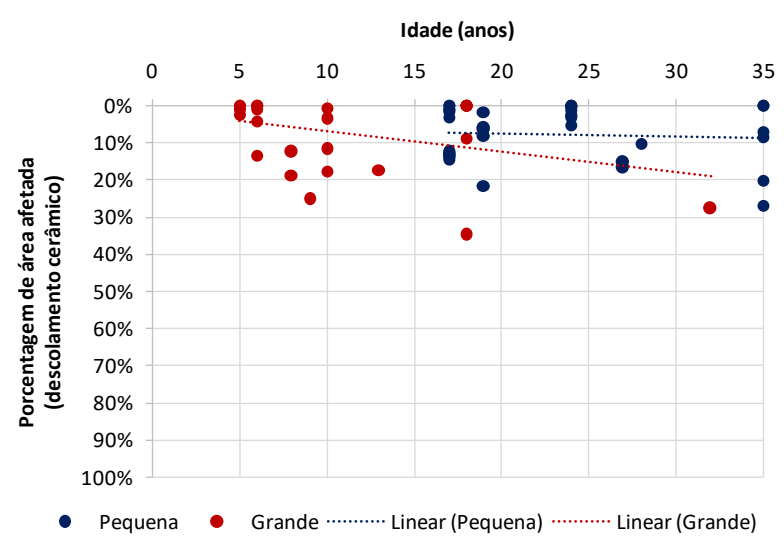

(c) Leste

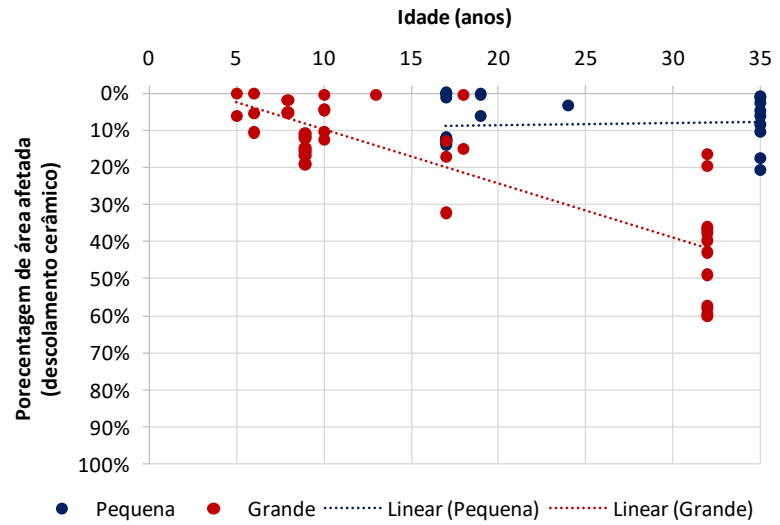

(b) Sul

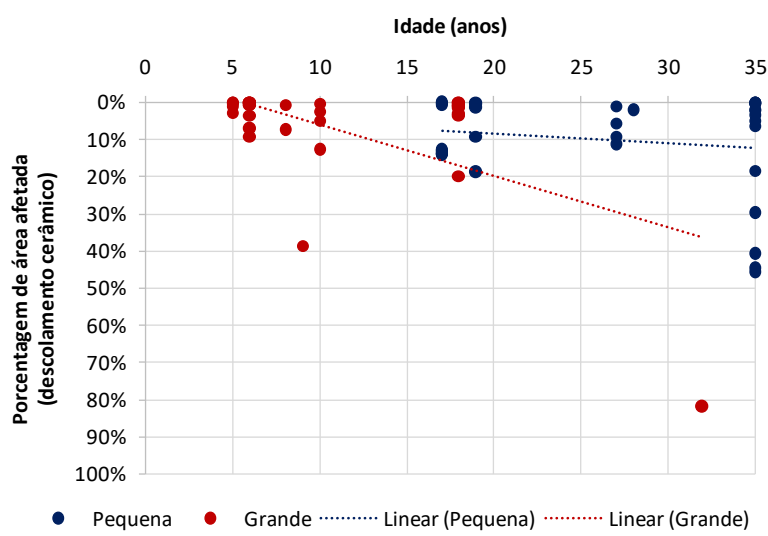

(d) Oeste

Figura 4 - Relação da porcentagem de área de descolamento cerâmico em função da dimensão da cerâmica para as orientações

Analisando as ocorrências de descolamento cerâmico, observa-se que as cerâmicas grandes apresentam maiores porcentagens de área com descolamento cerâmico para todas as orientações de fachada. Para as fachadas Sul e Oeste, as cerâmicas pequenas apresentaram as menores porções de áreas com deslocamento cerâmico relativamente às cerâmicas grandes. Essa situação pode ser explicada pelos baixos níveis de radiação solar recebida por essas orientações ao longo do ano, assim impedindo que as cerâmicas pequenas sofressem maiores degradações associadas à radiação solar, evidenciando a maior dificuldade que as cerâmicas grandes enfrentam para dissipar tensões internas, como afirmado por Uchôa (2015).

Além da função estética, os rejuntes apresentam funções de proteção contra a umidade, assim evitando a penetração de água e até impedindo que as peças descolem da argamassa. A Figura 5 ilustra a relação da porcentagem de área de falha no rejunte ao longo do tempo em função da dimensão das cerâmicas para as orientações. 


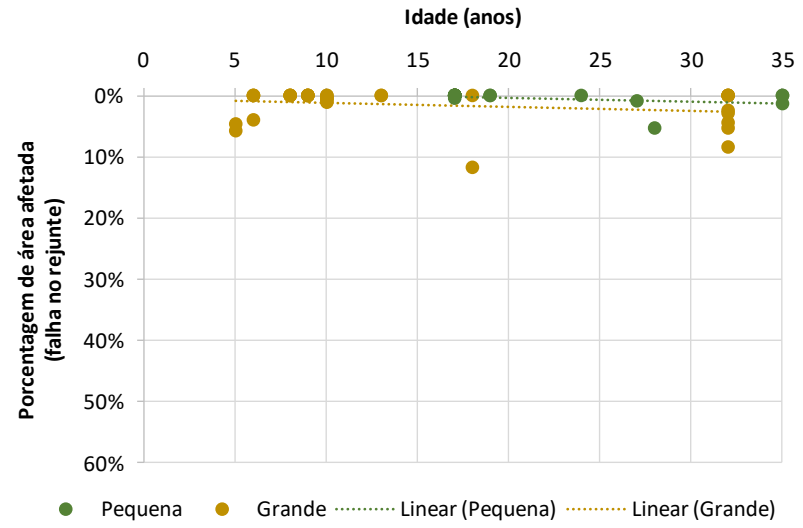

(a) Norte

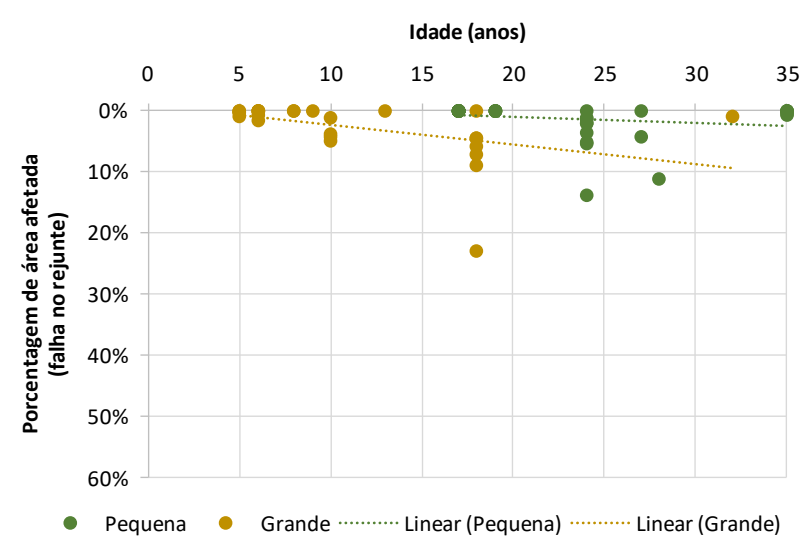

(c) Leste

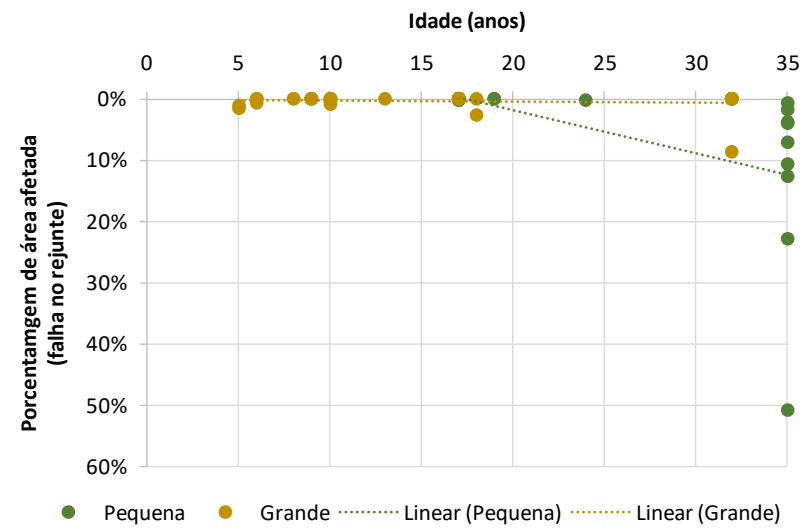

(b) Sul

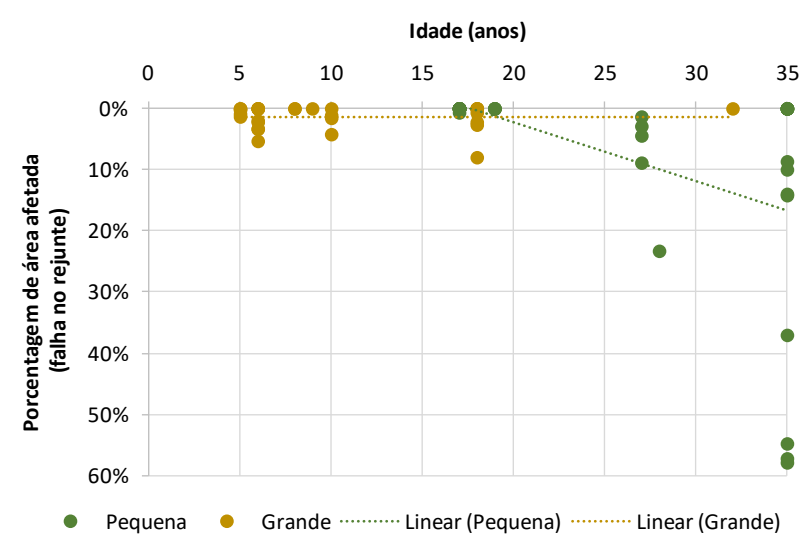

(d) Oeste

Figura 5 - Relação da porcentagem de falha no rejunte em função da dimensão da cerâmica para as orientações

$\mathrm{Na}$ análise de falha no rejunte, os resultados apontaram as fachadas revestidas por cerâmicas grandes com maiores áreas de falha no rejunte orientadas para o Norte e para o Leste. Em contra partida, as fachadas orientadas ao Sul e a Oeste revestidas por cerâmicas pequenas apresentaram maiores porções de área com falha no rejunte. Uma vez que há uma pequena quantidade de fachadas com falha no rejunte tanto para as cerâmicas grandes quanto para as pequenas, esses resultados devem ser observados com parcimônia, a fim de se evitar conclusões precipitadas.

Ainda assim, semelhante a análise da claridade da cerâmica, as maiores porcentagens de área afetada por falha no rejunte nas cerâmicas grandes em fachadas orientadas ao Norte e a Leste podem ser explicadas pelo fato de essas orientações sofrerem maiores incidências de radiação solar ao longo do ano. Assim, como afirmado por James et al. (2001), cerâmicas grandes estão sujeitas a maiores tensões e deformações vide as maiores dilatações térmicas que sofrem por causa da direta proporcionalidade entre a expansão térmica e a dimensão do material.

Por outro lado, as cerâmicas pequenas com maiores áreas com falha no rejunte nas fachadas orientadas ao Sul e a Oeste podem ser justificadas pela menor exposição devido às baixas taxas de radiação solar recebidas anualmente. Assim sendo, a dilatação térmica não é a principal causadora de falha no rejunte, mas sim a retração térmica.

\section{Conclusões}

Esta pesquisa traz como principal contribuição a análise de características da fachada que refletem no comportamento da degradação de fachadas revestidas por cerâmicas. A anomalia referente ao descolamento cerâmico é destacada por ser a anomalia mais incidente e estar associada a uma elevada gravidade podendo causar risco ao usuário. Diversas características das fachadas foram testadas, com os atributos associados ao material, claridade e dimensão da cerâmica, apresentando maior força de 
correlação com o FGD. As condições de exposição associadas aos agentes externos de degradação são fortemente relacionados às causas das anomalias, principalmente quando é considerado a ação da radiação solar ao longo do ano. Baseados nos resultados encontrados, é recomendado o uso de pastilhas pequenas e claras nas fachadas, principalmente nas fachadas orientadas ao Norte. Considerando as análises das caraterísticas de projeto e da exposição das fachadas fica evidente a importância da etapa de projeto na degradação e, consequentemente, na vida útil do sistema de revestimento de fachada.

\section{Agradecimentos}

Os autores agradecem o apoio da Universidade de Brasília e da Universidade de Lisboa e a disponibilização de informações pelo grupo de pesquisa do Programa de Pós-Graduação em Estruturas e Construção Civil da Universidade de Brasília que investiga degradação em fachadas. Ademais, agradecem à Fundação de Apoio à Pesquisa do Distrito Federal (FAPDF) pela bolsa de estudo e pesquisa concedida.

\section{Referências Bibliográficas}

Antunes, G. R. (2010) Estudo de manifestações patológicas em revestimento de fachada em Brasília: sistematização da incidência de casos. Dissertação de Mestrado. Universidade de Brasília. Brasília, Brasil. $166 \mathrm{p}$.

Bauer, E., de Souza, J. S., \& Piazzarollo, C. B. (2020). Application of the Degradation Measurement Method in the Study of Facade Service Life. In Building Pathology, Durability and Service Life (pp. 105-119). Springer, Cham.

Bauer, E.; Castro, E. K.; Silva, M. N. B. (2015) Estimativa da degradação de fachadas com revestimento cerâmico: estudo de caso de edifícios de Brasília. Cerâmica. v. 6. pp. 151-159.

Bauer, E; Castro; E. K.; Silva, M. N. B.; Zanoni, V. A. G. (2014) Evaluation of damage of building facades in Brasília. International Conference on Durability of Building Materials and Components - DBMC. São Paulo, Brasil. pp. 535-542.

Bordalo, R.; de Brito, J.; Gaspar, P. L.; Silva, A. (2011) Service life prediction modelling of adhesive ceramic tiling systems. Building Research and Information. v. 39. n. 1.pp.66-78.

Braga, D. K.; Amorim C. N. D. Conforto térmico em edifícios residenciais no Plano Piloto de Brasília. In: claCS ENTAC 04 - Construção Sustentável, 2004, São Paulo. I Conferência Latino Americana de construção sustentável e X ENTAC - Encontro Nacional de Tecnologia do Ambiente Construído, 2004. v. 1.

Corbella, O. D.; Yannas, S. Em Busca de uma Arquitetura Sustentável para os Trópicos. 2ª ed. Rio de Janeiro: REVAN, 2009. v. 1. 308p.

Daniotti, B.; Re Cecconi, F. (2010) Test Methods for Service Life Prediction-State of the Art Report on Accelerated Laboratory Test Procedures and Correlation between Laboratory Tests and Service Life Data. W080-Prediction of Service Life of Building Materials and Components. pp. 220-207.

Francinete Jr P.; Souza J.S.; Zanoni V.A.G; Bauer E.; Silva M.N.B. Relação entre a degradação de fachadas e a incidência de chuva dirigida e temperatura - estudo de caso para os edifícios de Brasília-Brasil. CONPAT 2015, Lisboa, Portugal, 2015.

ISO 15686-7 (2012) Buildings and constructed assets - Service life planning - Part 7: Performance evaluation for feedback of service life data from practice. International Organization for Standardization.

James, J. D.; Spittle, J. A.; Brown, S. G. R.; Evans, R. W. (2001) A review of measurement techniques for the thermal expansion coefficient of metals and alloys at elevated temperatures. Measurement science and technology. v. 12. n. 3, pp. 1.

Lacasse, M. A. (2003) Durability and performance of building envelopes. BSI 2003 Proceedings. pp. 1-6.

Moscoso, Y.F.M. (2013) Estudo numérico e experimental de tensões atuantes na argamassa colante de fachadas de edificações sob ação da fadiga termo-mecânica. Dissertação de Mestrado, Universidade de Brasília, Brasília, 2013, 142p. 
Piazzarollo, C. B. (2019) Estudo da evolução e da gravidade da degradação nas diferentes zonas componentes da fachada. Dissertação de Mestrado. Universidade de Brasília. Brasília, Brasil. 102 p.

Pinheiro, P. I. S. (2016) Aplicação do Método de Mensuração da Degradação (MMD) ao estudo das fachadas de edifícios em Brasília. Trabalho de conclusão de curso. Bacharelado em Engenharia Civil. Universidade de Brasília. Brasília, Brasil. 186 p.

Ribeiro, F. A.; Barros, M. M. S. B. (2010). Juntas de movimentação em revestimentos cerâmicos de fachada. Pini, São Paulo.

Shohet I.M.; Laufer A. (1996) Exterior cladding methods: a techno economic analysis. Journal of Construction Engineering and Management, 122(3). pp. 242-247.

Silva, M. N. B. (2014) Avaliação quantitativa da degradação e vida útil de revestimentos de fachada Aplicação ao caso de Brasília/DF. Tese de Doutorado. Universidade de Brasília, Brasília.

Souza, J. S. (2016) Evolução da degradação de fachadas - efeito dos agentes de degradação e dos elementos constituintes. Dissertação de Mestrado. Universidade de Brasília, Brasília, Brasil. 114p.

Souza, J. S. (2019) Impacto dos fatores de degradação sobre a vida útil de fachadas de edifícios. Teste de Doutorado. Universidade de Brasília, Brasília.

Souza, J., Silva, A., de Brito, J., \& Bauer, E. (2018a). Application of a graphical method to predict the service life of adhesive ceramic external wall claddings in the city of Brasília, Brazil. Journal of Building Engineering, 19, 1-13.

Souza, J., Silva, A., de Brito, J., \& Bauer, E. (2018b). Service life prediction of ceramic tiling systems in Brasilia-Brazil using the factor method. Construction and building materials, 192, 38-49.

Uchôa, J. C. B. (2015) Análise numérica e experimental da fadiga termomecânica em argamassas colantes no sistema de revestimento cerâmico. Tese de Doutorado. Universidade de Brasília, Brasília. 